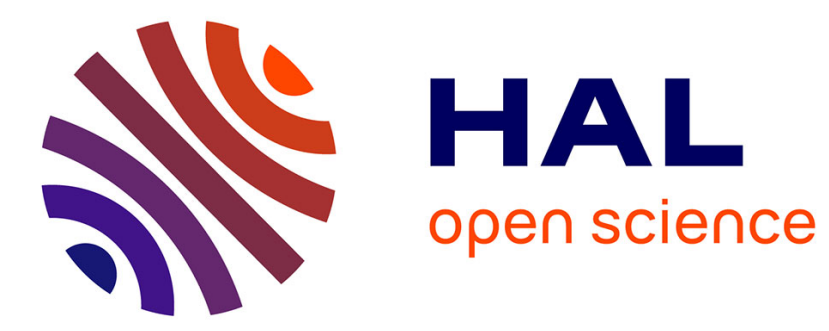

\title{
Electromagnetic Time Reversal in the Near Field: Characterization of Transient Disturbances in Power Electronics
}

Sassia Hedia, Bessem Zitouna, Jaleleddine Ben Hadj Slama, Lionel Pichon

\section{- To cite this version:}

Sassia Hedia, Bessem Zitouna, Jaleleddine Ben Hadj Slama, Lionel Pichon. Electromagnetic Time Reversal in the Near Field: Characterization of Transient Disturbances in Power Electronics. IEEE Transactions on Electromagnetic Compatibility, 2020, 62 (5), pp.1869-1878. 10.1109/TEMC.2020.2965735 . hal-02522867

\section{HAL Id: hal-02522867 \\ https://hal.science/hal-02522867}

Submitted on 28 Jul 2020

HAL is a multi-disciplinary open access archive for the deposit and dissemination of scientific research documents, whether they are published or not. The documents may come from teaching and research institutions in France or abroad, or from public or private research centers.
L'archive ouverte pluridisciplinaire HAL, est destinée au dépôt et à la diffusion de documents scientifiques de niveau recherche, publiés ou non, émanant des établissements d'enseignement et de recherche français ou étrangers, des laboratoires publics ou privés. 


\title{
Electromagnetic Time Reversal in the Near Field: Characterization of Transient Disturbances in Power Electronics
}

\author{
Sassia Hedia, Bessem Zitouna, Jaleleddine Ben Hadj Slama, and Lionel Pichon
}

\begin{abstract}
In the present paper, the electromagnetic time reversal technique (EMTR) is studied in the electromagnetic compatibility (EMC) context. The main issue addressed by this approach is to deal with EM radiating sources identification. This paper is intended to provide a time domain (TD) study of EMTR in the near field (NF) and prove its efficiency in characterizing transient disturbances in power electronics. The reconstruction of EM emissions is based on two stages. First, modeling process relies on the use of TD analytical expressions. Second, signal processing is referring to time reversal (TR) technique, which defines an inverse problem resolution. The method proposes to identify a set of equivalent dipoles. The validation is carried out experimentally using a TD measurement bench. The main purposes of this method is to extract the dipoles locations, moments and orientation. The magnetic field maps calculated at each time step using obtained equivalent sources are compared to measured distributions. Adequate agreement is achieved, which confirms the efficiency of the proposed method. In addition, for validation purposes, the equivalent model is compared to that obtained by a frequency domain (FD) method based on the standard genetic algorithms. Unlike FD, TD investigations allow characterizing transient disturbances in power electronic systems that emit strong EM interferences, by finding a sufficient equivalent model valid on a wide frequency band at once.
\end{abstract}

Index Terms - Electromagnetic time reversal (EMTR), time domain (TD) analysis, near-field (NF) technique, radiated emissions, transient perturbation.

\section{INTRODUCTION}

E LECTRONIC devices are increasingly susceptible to severe electromagnetic interferences (EMI). This phenomenon might occur during switching phase in power electronics due to quickly changing current and voltage transients (i.e. a fast di/dt) [1-2]. Multiple research works have already been conducted to deal with these disturbances. The identification of emissive sources is required to investigate electromagnetic compatibility (EMC) compliance and characterize devices. Specifically, there has been a growing interest in studying radiated emissions [2-3]. Electromagnetic (EM) radiation can be measured in either far field or near field (NF). However, NF measurement has interesting advantages in terms of cost, accuracy, and application range. Moreover, NF scanning technique is less dependent on test conditions. Thus, it has been used for obtaining EM fields, providing EMC studies and sources diagnostics. For instance, unlike the far field technique, it can be performed to estimate the current distribution on microstrip traces in a printed circuit board in the high frequency or for faults locations [3-4]. In order to evaluate EM radiation in the near field region, modeling methods based on inverse problem resolution have been widely presented in the literature [3], [5-11]. Indeed, EMC NF measurements for inverse problems are performed in the reactive region and are generally interested in the identification of real or equivalent radiating sources in the studied structure [3], [5-14].

In this regard, the EM inverse problem has been first established in the frequency domain (FD) to estimate radiated disturbances, using a set of equivalent dipoles [2], [5-9]. For instance, several works have proposed a matrix inversion method to obtain equivalent parameters, which needs a linear system resolution [5-7]. Despite its efficiency, this method needs a large set of measurements and leads to several conditioning problems. Thus, optimization methods, namely genetic algorithms (GA), have been introduced [8-9] to reduce the number of dipoles and obtain a 3D model of the device under test (DUT), but this process needs a heavy computational time. Therefore, authors in [9] have investigated the use of hybrid methods, based on GA, pseudo zernike moment invariant (PZMI), artificial neural networks (ANN), in order to avoid previous limitations and speed up the convergence of the algorithm at a fixed operating frequency. However, it is well known that power electronic systems emit non-sinusoidal EM radiations of short duration, especially in the transient regime were radiation strongly disturb the environment. In certain cases of transient emissions, analyses only in the FD are insufficient [10-11]. Hence, to overcome this issue, researchers have recently moved towards the investigation of EM inverse methods in the TD [10-16].

In [11], a GA-based method has been developed in TD, but it is sensible to selected GA options. In [12-14], authors have demonstrated the efficiency of TD methods to deal with EM transients that are emitted by a micro strip device. Nevertheless, in these approaches the main drawback has been the limitation in terms of time and space resolutions, which mainly depends on the frequency range and on the use of powerful calculating machines [12]. Therefore, alternative methods based on the electromagnetic time reversal (EMTR) have been proposed in [15-16] using TD analysis. Indeed, the naming of EMTR is dedicated to the application of the time reversal (TR) technique in the field of electromagnetics. TR has been used for many years in the literature mainly for source location identification [17-24]. Multiple studies and works have been achieved based 
upon the acoustic wave equation [17-19]. Recently, successful studies were led in electromagnetism, particularly in the EMC context and power systems [15-16], [20-24]. TR has shown several advantages, its straightforward applicability and robustness for inverse scattering or fault detection in complex medium [15], [18]. For instance, Rachidi et al. has investigated the relevance of EMTR in TD, notably for faults location in power networks [15]. Indeed, TR proceeds directly in the time domain and so is well suited to transient fields. For instance, TR approaches presented in [16], [25] and [26] are applied in case of band-limited signals in order to reconstruct transient sources. In these works, time reversal mirrors (TRM) are placed in the far-field region. This is a typical situation where TR is appropriate. Recent works [19], [27-29] investigated time reversal technique in the near field and demonstrated focal spots when the TRM is placed in the NF region of emissive sources.

The aim of this paper is to propose a characterization method in order to evaluate EM disturbances in the NF for power electronic systems. Indeed, in structures such as power converters, voltage and current commutations in nonlinear devices, essentially transistors, create critical non-intentional transient perturbations. In such situations, the transient sources cover a very wide frequency band. Thus, the determination of radiating sources is especially required to design magnetic shielding and reduce EM radiations. This includes sources parameters prediction, such as positions and orientations of dipoles moment that characterize the structure. In [23], the TD EMTR has been applied using a sinusoidal excitation signal in a simulated test. The proposed method has been validated, in [24], in the case of a Gaussian current in order to assimilate nonlinear systems behavior. In this paper, the EM inverse method based on TR is performed in TD using the NF technique in order to provide an adequate equivalent model that radiates the same emissions as the studied structure in case of several transient sources. Moreover, for validation purposes, a comparative study between the EMTR-based method in the time domain and a conventional inverse method in the frequency domain has been performed using GA as an optimization method.

Within this context, the present paper is organized as follows: Section II states the principle of EMTR method using TD identification. In section III, the proposed EMTR-based method is applied to a simulation test case. Synthetic current loops are used to create radiated magnetic field. A virtual TRM is defined in order to perform the back propagation of timereversed waveforms. The experimental validation is carried out in Section IV. Conclusion is presented in Section V.

\section{EMTR-BASED DISTURBANCES IDENTIFICATION IN TD}

\section{A. Time Reversal Technique}

Time reversal theory has been applied for a long time in acoustics and ultrasounds applications [17-19]. Recently, this technique has had a tremendous success in the wide field of electrical engineering [15-16], [20-24]. The originality of TR is to offer the possibility of focusing a wave in both space and time. Overall, TR technique is founded on the following steps:

a) Forward propagation and measurement: a short pulse illuminates a source. Emitted signals propagate in the medium, and are then recorded at the time reversal mirror, which is a set of receiving transducers.

b) Time reversing and back-propagation: time-reversed waves are re-emitted in the same model as the first step.

c) Focusing: the source location is obtained at a proper time step. Back-propagated signals combine constructively at the real source position to reach a maximum.

Thanks to the reciprocity theorem, the same field is acquired by emitting a pulse at the TRM and recording the signal at a source point without alteration. Indeed, the back-propagation is based on the reversibility in time of the wave equation.

Typically, TR technique is based on the DORT method, which is the French acronym for decomposition of the time reversal operator. This method depends on the determination of the TR invariants (TRI) of the TR operator (TRO) in FD [1718]. However, the standard DORT has produced a deficiency when dealing with multiple sources emissions simultaneously. Thus, in order to overcome the shortcomings of DORT method, the time domain analysis has been introduced to enlarge the TR validity and increase its robustness. Indeed, at one frequency, TRM behaves like a phase conjugate mirror (PCM), allowing the transition to the TD [15-16], [25].

\section{B. Applying EMTR Principle}

TR has been proven to be capable of providing a solution to spatial and temporal reconstruction problems and locating sources [15-24]. The present work aims to investigate the use of the TR technique for an EMC application, thereby retrieving emissive sources using radiated magnetic field over a period $T$.

As explained previously, TR leans on the principle of reciprocity. Particularly, regarding electromagnetic wave equation, the invariance under TR can be exploited for focusing EM signals. The wave equation in electromagnetics, which is a homogeneous equation, can be written as follows:

$$
\frac{1}{c^{2}} \frac{\partial^{2} \Phi}{\partial t^{2}}=\nabla^{2} \Phi
$$

where $\Phi$ stands for magnetic $\mathrm{H}$ or electric $\mathrm{E}$ field, and c represents the speed of light in free space. Indeed, if $f(t)$ is a solution of equation (1), there exist another solution to (1), which is $g(t)=f(-t)$. Hence, theoretically, an EM wave can propagate backward from final time $t=\mathrm{T}$ to $t=0 \mathrm{~s}$. In practice, assuming $H(r, t)$ the magnetic field measured by TRM at probe position $r$ at each time step $t$. The backpropagated radiated field is $H(r, \mathrm{~T}-t), t \in[0 ; \mathrm{T}]$. It is noted that under the action of time inversion, the radiated field $H$ is of an odd parity [15], [30]. Consequently, if we consider 'TR' the time inversion operator given by $\operatorname{TR}\{\Phi(r, t)\}=\Phi(r,-t)$, we have the relation:

$\operatorname{TR}\{\mathrm{H}(r, t)\}=-\mathrm{H}(r,-t)$

Let us consider $I(t)$ the current flowing through the radiating source and suppose that we have $\mathrm{N}_{\mathrm{a}}$ receiving antennas, forming the TRM, placed above the DUT. Thus, as in [16] and [25], measured magnetic field $H$ is a function of $I(t)$ and can be written as:

$$
\mathrm{H}_{i}(t)=h\left(r_{0} \rightarrow r_{i}, t\right) \otimes \mathrm{I}(t)
$$


where $\otimes$ denotes a convolution product, $1 \leq i \leq N_{a}$, and $h\left(r_{0} \rightarrow r_{i}, t\right)$ is the impulse response of the system at a measuring position $r_{i}$, for a pulse emitted from source $r_{0}$. Hence, $h(t)$ is a transfer matrix that describes transmit-receive process performed between the array of $\mathrm{N}$ receivers (TRM antennas) and the array of $\mathrm{M}$ transmitters (virtual network). Therefore, each transmitting element $\mathrm{M}$ and receiving element $\mathrm{N}$ are linked by an inter-element impulse response calculated analytically using a delta function as excitation signal. Thereafter, time-reversed magnetic field is re-injected from $r_{i}$. Focused signal in $\mathrm{r}_{0}$ are instantaneously defined as in [25]:

$$
\mathrm{H}_{\mathrm{TR}}\left(r_{0}, t\right)=\sum_{\mathrm{i}=1}^{\mathrm{N}} h\left(r_{i} \rightarrow r_{0}, t\right) \otimes \mathrm{H}_{\mathrm{x}, \mathrm{y}, \mathrm{z}_{\mathrm{i}}}(-t)
$$

where $\mathrm{H}_{\mathrm{TR}}\left(r_{0}, t\right)$ stands for focused field at source location $\mathrm{r}_{0}$ and focusing time $t$. Thus, the absolute maximum of focused signal, during the period $\mathrm{T},[25]$, is expressed as follows:

$$
\operatorname{Max}\left(r_{0}\right)=\max _{t \in \mathrm{T}}\left(\left|\mathrm{H}_{\mathrm{TR}}\left(r_{0}, t\right)\right|\right)
$$

In the following, a particular care is given to time and space focusing. Before going further, we need to recall some major acquirements from TR theory. It has been shown in the literature that the obtained focusing signal matches with the reversed excitation shape [16], [20-21], [25]. In addition, in this work, we suppose that the magnetic field is approximately proportional to the current flowing through the structure.

\section{EM Transient Modeling Using Equivalent Magnetic Dipoles}

In the NF region, the field properties are determined by the source characteristics. For instance, power electronic circuits are characterized by the magnetic field predominance. In order to provide an efficient equivalent model of the studied structure, the investigation of elementary dipoles, such as current loops, is required for the inverse problem resolution in the near field zone of EM radiation. Indeed, obtained equivalent dipoles refer to an analytical model that has the same radiation behavior as the real DUT. An elemental magnetic dipole, Fig.1, is defined using the center coordinates $\left(X_{d}, Y_{d}, Z_{d}\right)$ and the orientation angles $(\theta, \varphi)$ of the moment [31]. This current loop, of surface $S=\pi \times r^{2}$, where $r$ is the radius, has a magnetic dipole moment $M_{d}$, defined as follows:

$$
\overrightarrow{\mathbf{M}_{\mathrm{d}}}=I \times \overrightarrow{\mathbf{S}}
$$

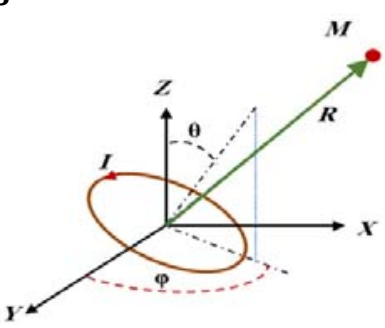

Fig. 1. Equivalent magnetic dipole (current loop).

In the near field, TD analytical equations of dipole radiation have been presented in [11], [24]. These expressions provide the magnetic emissions of components $H_{x}, H_{y}$ and $H_{z}$, at the measurement point $\mathrm{M}\left(X_{0}, Y_{0}, Z_{0}\right)$, as follows:

$$
\mathrm{H}_{x, y, z}=A\left[\left(B_{1} C_{x, y, z}\right)-\left(B_{3} D_{x, y, z} E\right)\right]
$$

- $A=\frac{1}{4 \pi R}$

- $B_{m=1,3}=\left(\left(\frac{1}{c^{2}} \frac{\partial^{2} M_{d}\left(t^{\prime}\right)}{\partial t^{2}}\right)+\left(\frac{m}{c R} \frac{\partial M_{d}\left(t^{\prime}\right)}{\partial t}\right)+\left(\frac{1}{R^{2}} M_{d}\left(t^{\prime}\right)\right)\right)$

- $C_{x, y, z}=(\sin (\theta) \cos (\varphi), \sin (\theta) \sin (\varphi), \cos \theta)$

- $D_{x, y, z}=\frac{1}{R^{2}}\left(\left(X_{d}-X_{0}\right),\left(Y_{d}-Y_{0}\right),\left(Z_{d}-Z_{0}\right)\right)$

- $E=\left(\begin{array}{c}\cos (\theta)\left(Z_{d}-Z_{0}\right)+ \\ \sin (\theta) \sin (\varphi)\left(Y_{d}-Y_{0}\right)+ \\ \sin (\theta) \cos (\varphi)\left(X_{d}-X_{0}\right)\end{array}\right)$

- $\mathrm{R}=\sqrt{\left(X_{d}-X_{0}\right)^{2}+\left(Y_{d}-Y_{0}\right)^{2}+\left(Z_{d}-Z_{0}\right)^{2}}$

- $t^{\prime}=t-\frac{R}{\mathrm{c}}$

\section{APPLICATION OF THE TD EMTR TEChNIQUE}

\section{A. Proposed Method}

The aim of the present study is to characterize EM emissions by building an equivalent radiation model of the studied structure using the NF scan. An EMTR-based algorithm is proposed to locate disturbances and identify instantaneous magnetic field waveforms using TD measurements.

Assume a radiating source excited by a transient current. Radiated fields are measured by a set of transducers acting as a TRM. Time-reversed measured signals are re-injected in order to back-propagate, focus and converge towards the location domain. It is worth noting that the transfer matrix is obtained analytically as in (7) using a virtual array of potential sources network arranged on the surface of the DUT and excited by a delta-function current. Indeed, the proposed method depends mainly on the EM radiation peak values extracted from the contributions of each active source along time. Once the most radiating sources are determined and their corresponding parameters and moment waveforms are extracted, using equations (4) and (5), reconstructed magnetic field signals are calculated in TD using (7). An error distribution is defined as the absolute difference between measured and estimated radiated field maps using identified dipoles. The method converges when the value of the obtained error is inferior to a predefined value assigned as a threshold. In the ideal case, the threshold equals to zero, which means that the estimated model provides the same radiation as the reference model. Otherwise, using experimental measurements, when the magnetic field value in the remaining map becomes inferior to the magnitude of the measurements noise, the process of identifying a further radiating source will stop. In this case, the threshold value should not be less than measurements errors, because we risk finding a model that does not have a physical sense. Knowing that measurements error can be obtained when making measurements without load. The flowchart of the proposed method is presented in Fig. 2. A preliminary validation has been performed in [23-24].

where: 


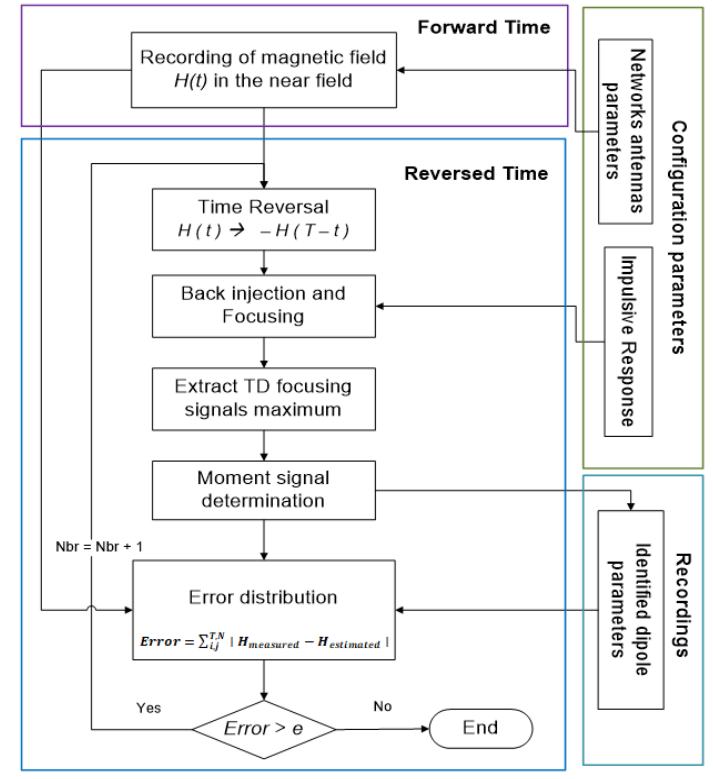

Fig. 2. Flowchart of the proposed method.

\section{B. Simulation Test}

In order to reproduce a behavioral model similar to EM radiation in power systems, the proposed method has been first applied to a simulation test. The algorithm is able to extract sources locations, orientations and moments. In this case, we consider two dipoles having different moment waveforms, Fig.3. Configuration parameters are presented in Table I. Calculated $\mathrm{H}_{x, y, z}$ maps are presented at $z=0$, in Fig. 4.a.

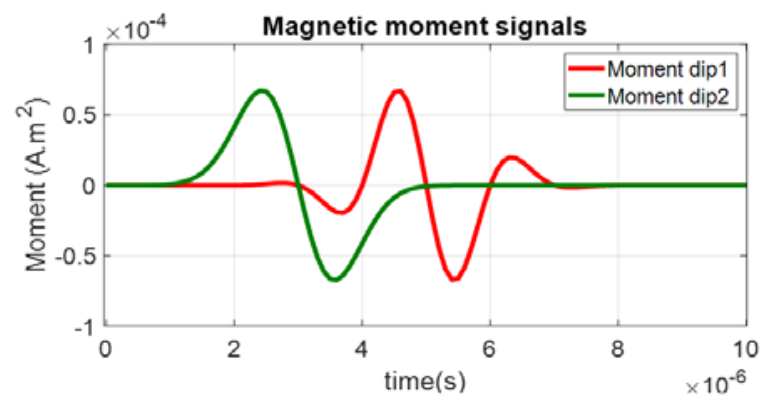

Fig. 3. Initial moment signals of dipoles 1 and 2.

TABLE I

CONFIGURATION PARAMETERS

\begin{tabular}{cc}
\hline \hline Simulation test & $(10 \mathrm{~cm} \times 10 \mathrm{~cm})$ \\
\hline Scanning area & $3 \mathrm{~cm}$ \\
Height of measurement & $2 \mathrm{~mm}$ \\
Scanning resolution & $(51 \times 51)$ \\
TRM antennas & $(21 \times 21)$ \\
Virtual sources network & $(1.5,1.5,0,1.5708,0)$ \\
Dipole 1 & $(-2,-2,0,0,0)$ \\
Dipole 2 & $(t)=M_{d 1_{0}} \frac{\left(t_{1}-t\right)}{\tau_{1}} \exp \left(\frac{-\left(t-t_{1}\right)^{2}}{\left(2 \tau_{1}\right)^{2}}\right)$ \\
Moment 1 & $M_{d_{1}}(t)$ \\
Moment 2 & $M_{d_{2}}(t)=M_{d 2_{0}} \sin \left(2 \pi f_{c} t\right) \exp \left(\frac{-\left(t-t_{2}\right)^{2}}{\left(2 \tau_{2}\right)^{2}}\right)$ \\
$\left(\tau_{1}, \tau_{2}, t_{1}, t_{2}, f_{c}\right)$ & $(0.4 \mu \mathrm{s}, 0.6 \mu \mathrm{s}, 3 \mu \mathrm{s}, 5 \mu \mathrm{s}, 0.5 \mathrm{MHz})$ \\
\hline \hline
\end{tabular}

Indeed, the first identified dipole corresponds to the most radiating area in the map. Thus, its parameters are immediately extracted. Looking at the error distribution, we note the presence of a further intense area. Therefore, the proposed method is carried out for another iteration. The maximum of the error map is compared at each iteration to a threshold value. When there is no other source to be found in the structure, the identification process stops. As a result, we find two magnetic dipoles as a number of radiating sources. The parameters of the obtained equivalent dipoles are shown in Table II. Moment maximum values were captured at $4.4 \mu \mathrm{s}$ for the first dipole and at $2.3 \mu$ s for the second one from a pulse duration of $10 \mu \mathrm{s}$.
Simulation

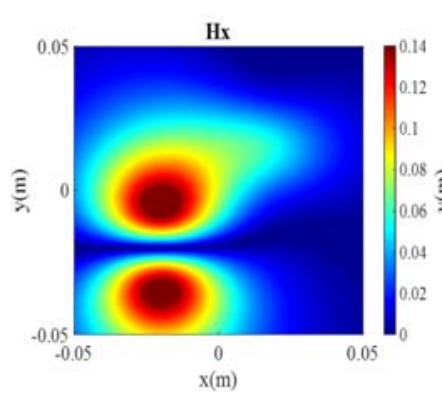

Hy

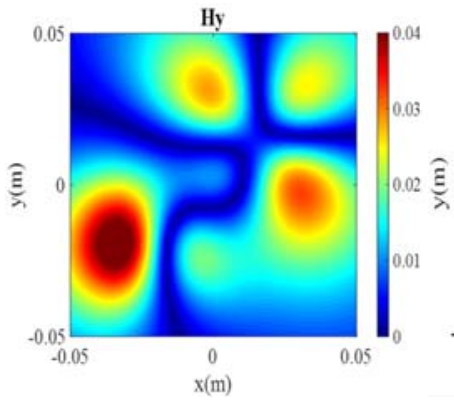

$\mathrm{Hz}$

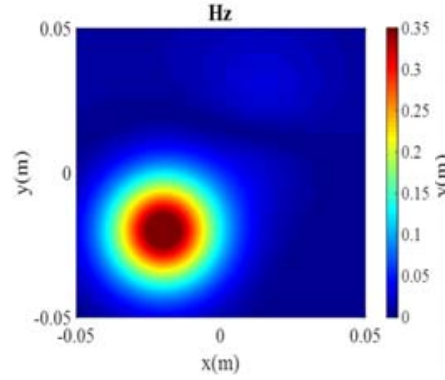

H

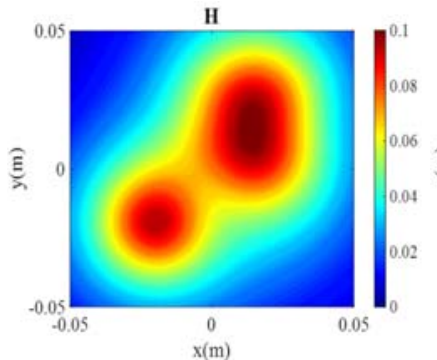

(a)
Proposed method
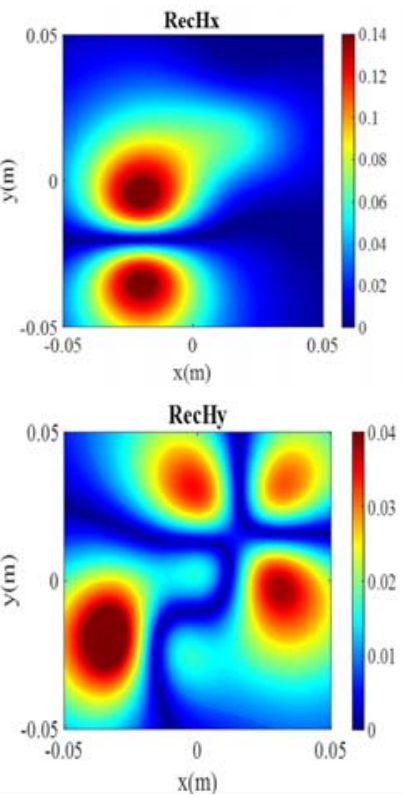

$\mathrm{RecHz}$

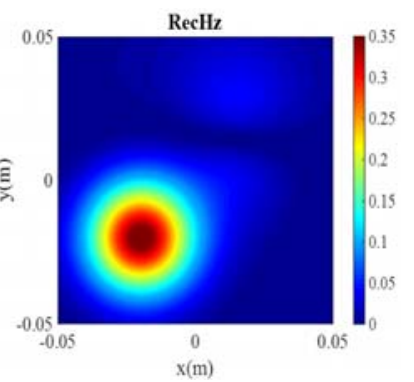

RecH

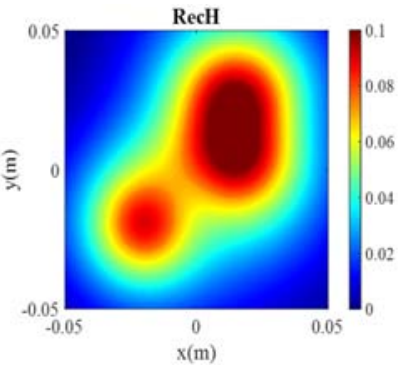

(b)
Fig. 4. (a) Calculated maps, (b) Estimated distributions (In $\mathrm{A} / \mathrm{m}$ ): $\mathrm{H}_{\mathrm{x}}$ at $t=$ $4.44 \mu \mathrm{s}, \mathrm{H}_{\mathrm{y}}$ at $t=4.24 \mu \mathrm{s}, \mathrm{H}_{\mathrm{z}}$ at $t=4.64 \mu \mathrm{s}$, Total field at $t=4.24 \mu \mathrm{s}$.

Estimated maps of magnetic field components, at $z=0$, are given in Fig. 4. b. The validation of sources positions in the location domain is presented in Fig. 5. Fig. 6 shows obtained moment signals. The first is a Gaussian pulse modulated by a 
sinusoid, whereas the second is a Gaussian derived signal. We notice an adequate agreement between simulated and estimated radiated field cuts along $x$-axis at $\mathrm{Y}=0$ and $z=0$, Fig. 7 .

TABLE II

Results - Identified parameters

\begin{tabular}{lccll}
\hline \hline & $\begin{array}{c}\text { Maximum moment } \\
\left(\mathrm{A} \times \mathrm{m}^{2}\right)\end{array}$ & $\begin{array}{c}\text { Position } \\
(\mathrm{m})\end{array}$ & $\begin{array}{c}\text { Orientation } \\
(\mathrm{rad})\end{array}$ & \\
\hline Dipole 1 & $6.684 \times e-05$ & $-0.02,-0.02,0$ & 0 & 0 \\
Dipole 2 & $6.642 \times e-05$ & $0.015,0.015,0$ & 1.5708 & 0 \\
\hline \hline
\end{tabular}

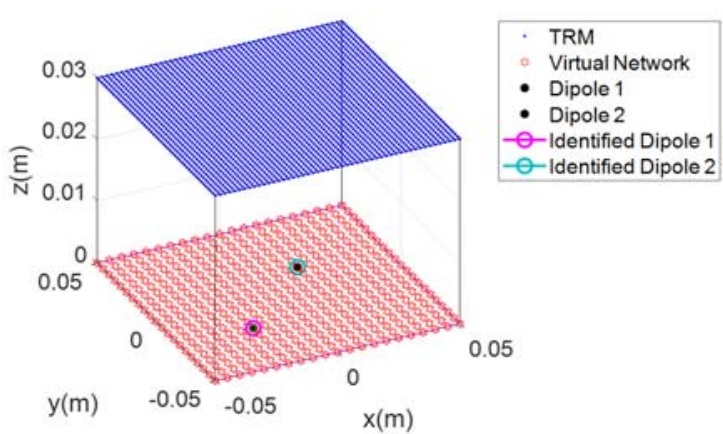

Fig. 5. Identification of real sources position.

Indeed, in Fig. 6, we observe a slight shift in amplitude and variances, especially at $t_{1}=2.9 \mu \mathrm{s}$ and $t_{2}=5 \mu \mathrm{s}$, when an overlap occurs. In fact, focusing relies on the calculation of a convolution product, which measures the amount of overlap between two signals. Hence, back-propagated and radiated fields will combine constructively at sources locations to reach a maximum. As long as sources are radiating simultaneously, focusing quality may be affected. These interferences have an impact on both signals, in the sense that when a dipole moment reaches a maximum, it affects the other dipole moment signal in the opposite direction.

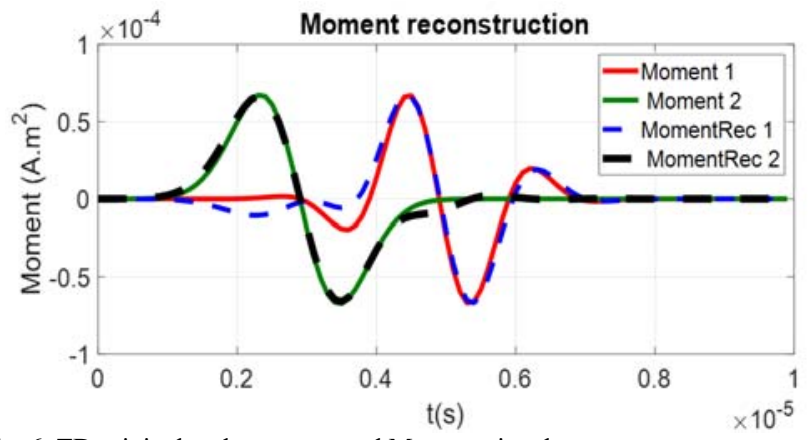

Fig. 6. TD original and reconstructed Moment signals.

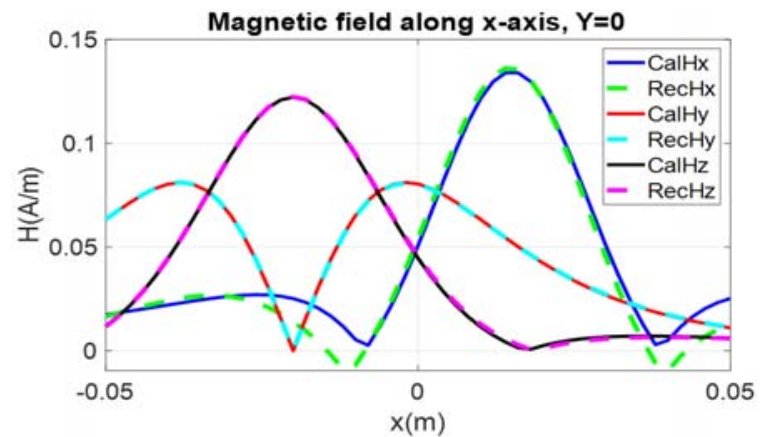

Fig. 7. Cuts of $\mathrm{H}_{\mathrm{x}, \mathrm{y}, \mathrm{z}}$ along $\mathrm{x}$-axis: Calculated and estimated at $\mathrm{t}=5 \mu$ s.

\section{EXPERIMENTAL VALIDATIONS}

\section{A. TD-based Measurement Test Bench}

Recently, adequate TD-based measurement tools such as high precision oscilloscopes have been presented in order to deal with EM emissions. It is worth noting that this bench is based on the NF scan. With the aim of making this solution suitable and useful for the characterization of transient disturbances, a synchronous acquisition is required. When the triggering event is detected, the sample is captured. Thus, synchronization is performed using a periodic and repetitive reference signal. Indeed, it is important to keep the same measurement conditions and prevent the impact of shifting from one scanning point to another (moving the probe) when making TD measurement. Measurement have been performed using a small coil probe as proposed and implemented in [12], Fig.8. The measuring probe, which is a magnetic loop of a radius equal to $1.6 \mathrm{~mm}$, generates a voltage from the varying magnetic flux. It is placed above the DUT in a way to avoid capacitive coupling and retain an acceptable signal to noise ratio. For the same reason, the probe is shielded and calibrated as in [12].

Since the principle of NF radiated emissions measurement in TD is quite similar to that carried out in the frequency domain, the used magnetic field probes can also be employed for TD measurements as a function of the considered frequency band. Generally, the major difference between the proposed TD measurement bench and the existing frequency one, [6-7],[32], is the replacement of the measured data receiver, which can be a vector network analyser (VNA) for amplitude and phase measurements or a spectrum analyser (SA) for amplitude measurement, by a high resolution oscilloscope. Indeed, the magnetic field is obtained by integrating the voltage measured at the terminals of the probe using the following equation: $\mathrm{H}(t)=-\frac{1}{\mu_{0} \times S} \int_{0}^{t} \mathrm{~V}(t) d t$, where $\mu_{0}=4 \times \pi \times 10^{-7} \mathrm{~N} / \mathrm{A}^{2}$, is the permeability in the free space and $S=\pi \times r^{2}$, is the probe surface $(r=1.6 \mathrm{~mm})$.

The probe is calibrated according to [12] with analytical formulas above a radiating structure whose radiation is theoretically known, at the same distance. Moreover, we have ensured that this shielded probe rejects very efficiently the electric field and measures the magnetic field with great accuracy. Input and output coaxial cables were also twisted and placed far apart from the measurement plane. Based on the mechanical displacement of measuring probe above the DUT in the NF region, the radiation field map is obtained instantaneously. A PC performs the acquisition of the measured datasets through the oscilloscope. Although this technique could be applied to a full three-dimensional configuration, we restrict the study for the scope of this paper to the normal component $\mathrm{H}_{\mathrm{z}}$. In order to validate the performance of the proposed method, an experimental test with a real circuit is carried out using a Lecroy WaveRunner 104XI oscilloscope. The proposed test bench is presented in Fig.8. 


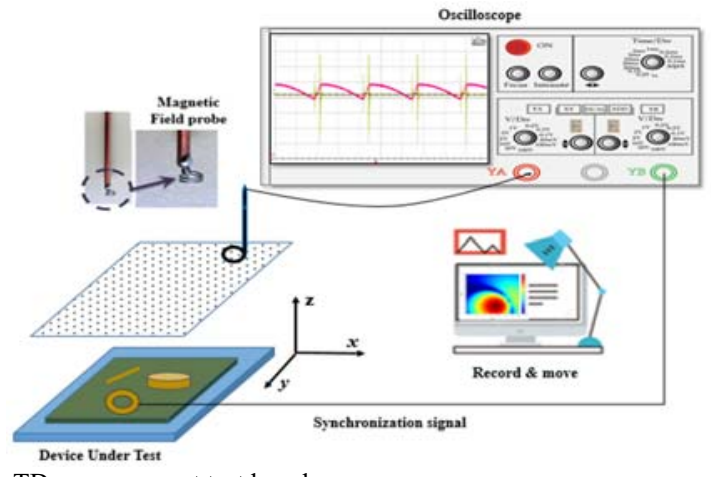

Fig. 8. TD measurement test bench.

\section{B. Experimental Test}

\section{1) Configuration of the Structure Under Study}

Switching converters are among major causes of EMI since they may carry fast changing di/dt events. For instance, a flyback circuit has several rapidly changing currents that need to be studied. In this work, the proposed method is applied to a flyback AC-DC converter based on the principle of switched-mode power supplies (SMPS), as in [33]. These power supplies present a high noise due to the switching frequency and can cause harmonic distortion. Hence, this type of converters may have some problems with regard to EMC. Furthermore, this device has both the characteristics of an industrial system with a complex structure and the characteristics of an academic circuit since it allows three different functions: Buck chopper, flyback power supply, and a forward power supply. Generally, this structure is used in low and medium power as in automotive lighting or battery charger. The flyback converter is equivalent to a buck-boost converter in performance and architecture. A flyback is a coupled inductor-based converter that stores energy in the magnetic circuit when current passes through and releases it when the power goes out. In the flyback configuration, the design of the circuit is of great importance in order to meet the converter specifications in terms of energy, magnetic storage and losses in materials.

In the practical test, a low power converter of $5 \mathrm{~W}$ is examined, see Fig. 9. The reason behind this choice is that this DUT is complex enough from an EMC point of view as long as it manifests transient over-voltages and oscillations that present different interferences modes covering a frequency band reaching several tens of $\mathrm{MHz}$. The schematic of the studied converter is presented in Fig. 10 and its characteristics are presented in Table V. Configuration parameters are also listed in Table V. Measurements were carried out for the normal component $\mathrm{H}_{\mathrm{z}}$ of the magnetic field, using the proposed TDbased measurement test bench, as described in Fig.10. Transient radiation signals at scanning points above the structure are measured with respect to a reference signal, Fig. 11 and Fig. 12. Indeed, using the schematic and the datasheet of the converter, the synchronization signal is chosen to be the signal on the input of the optocoupler, which is a periodic and a repetitive signal.
TABLE V

CONFIGURATION PARAMETERS

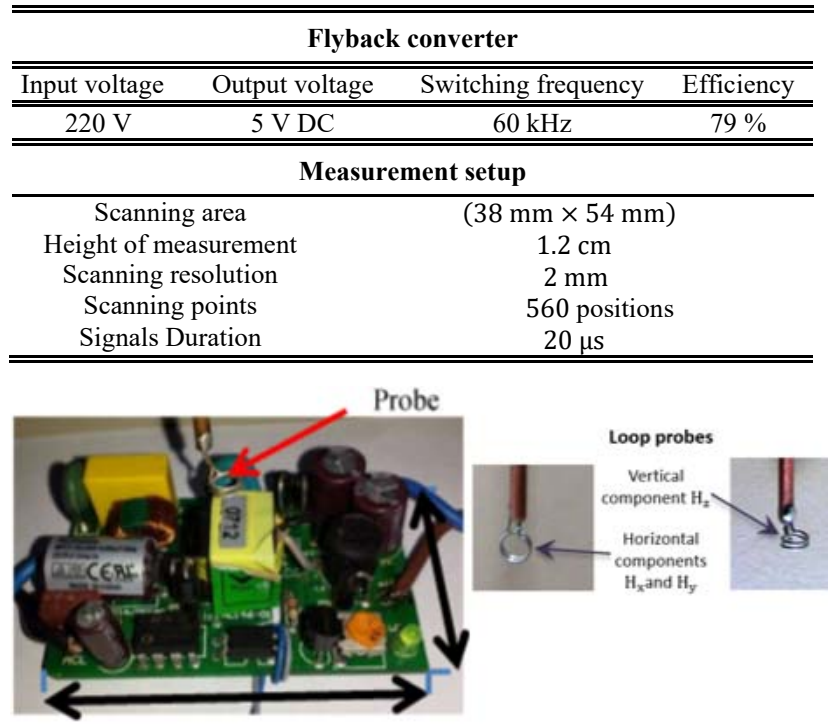

Fig. 9. Studied flyback AC/DC converter.

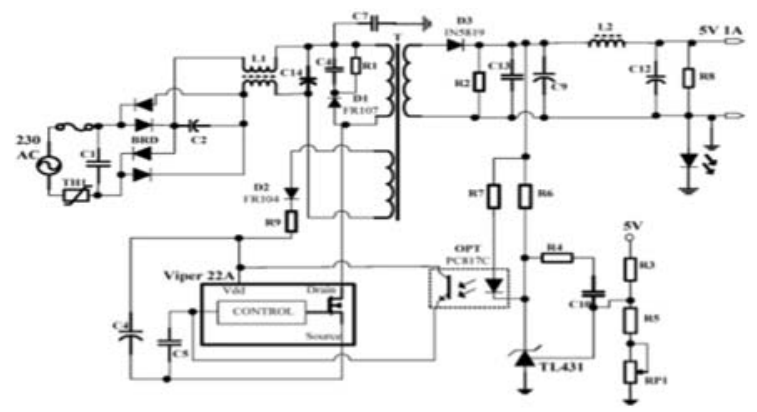

Fig. 10. Schematic of the studied flyback AC/DC converter.

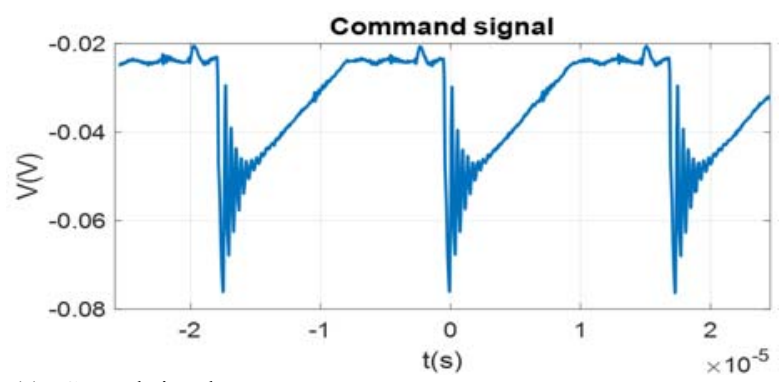

Fig. 11. Control signal.

a)

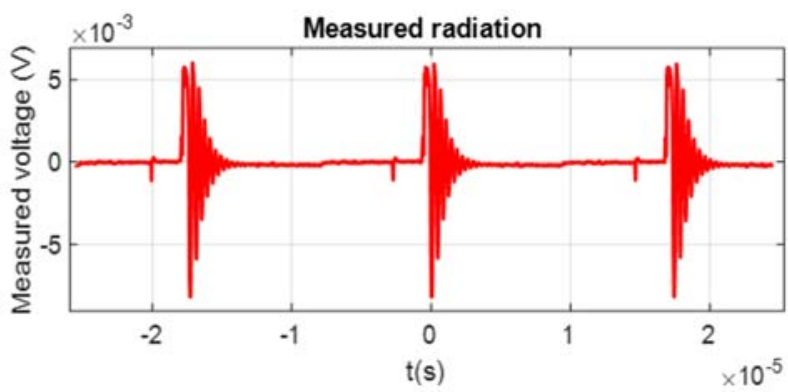


b)

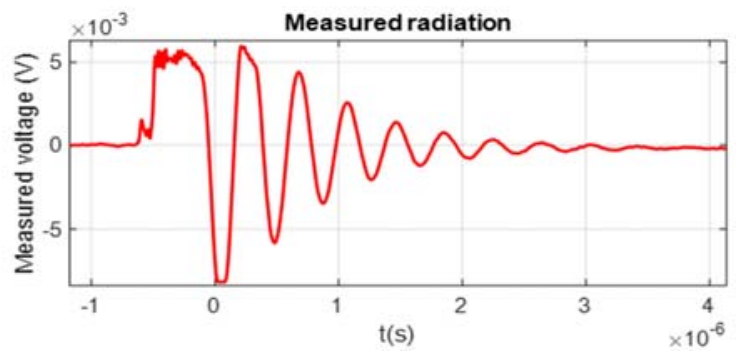

Fig. 12. A measured radiation signal: (a) Total signal, (b) Zoom.

In the studied system, we have noticed that sources are not radiating simultaneously all the time. For instance, an intense radiation area appears in the map of Fig. 13.a, and it does not exist in Fig. 13.b. and vice versa. The evolution of EM emissions happens over time. This fact is not considered using frequency analysis where all radiating elements appear together as if all sources are emitting at once, which is not very conform with power electronics radiation behavior.

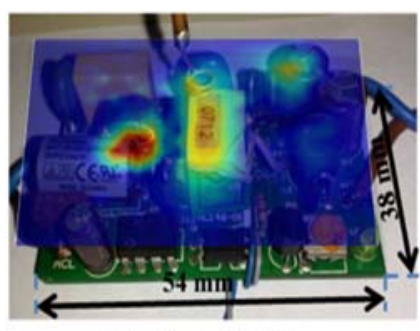

(a) at $t_{1}=10.25 \mu \mathrm{s}$

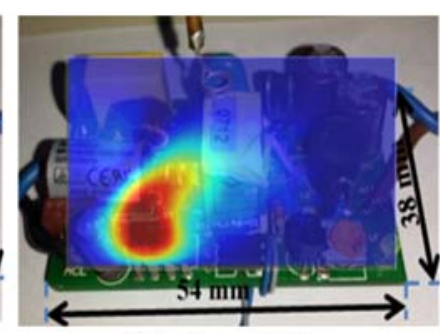

(b) at $t_{2}=10.5 \mu \mathrm{s}$
Fig. 13. Measured radiation field maps at different time steps.

\section{2) Application of the Proposed Method}

In order to identify an equivalent radiation model of the ACDC converter, the proposed method has been applied as discussed in section III.A. Thus, a virtual network of $(15 \times 15)$ potential sources is created to acquire the transfer matrix. Sources parameters of the obtained equivalent model are presented in Table VI. Indeed, in the studied converter, we have obtained seven magnetic dipoles. These identified sources correspond to different waveform shapes observed when scanning the board, Fig. 13. Based on the obtained equivalent model parameters, the radiation behavior of the DUT is reproduced along time. Estimated maps are compared to measured field distributions at several time steps randomly chosen. Fig. 14 shows the measured and the reconstructed $H_{z}$ field. Furthermore, Fig. 15 presents a comparison between estimated and measured radiated field cuts at $Z=0$.

TABLE VI

Model parameters

\begin{tabular}{ccccc}
\hline \hline & $\begin{array}{c}\text { Maximum Moment } \\
\left(\mathrm{A} \times \mathrm{m}^{2}\right) \times \mathrm{e}-7\end{array}$ & $\begin{array}{c}\text { Position } \\
(\mathrm{mm})\end{array}$ & $\begin{array}{c}\text { Orientation } \\
(\mathrm{rad})\end{array}$ \\
\hline$\# 1$ & 0.1545 & $10.9 ; 11.6 ;-0.67$ & 0 & 1,5708 \\
$\# 2$ & 0.2477 & $5 \mathrm{e}-3 ; 2 \mathrm{e}-2 ; 0$ & 0 & 0 \\
$\# 3$ & 0.0708 & $-2.7 ;-11.6 ;-0.7$ & 0 & $-1,5708$ \\
$\# 4$ & 0.0163 & $5.4 ;-15.4 ;-1.2$ & 1,5708 & 1,5708 \\
$\# 5$ & 0.09 & $13.6 ;-3.8 ;-0.37$ & 1.5708 & 0 \\
$\# 6$ & 0.0179 & $-5.4 ; 3.9 ; 0$ & 0 & 0 \\
$\# 7$ & 0.3468 & $13.6 ;-7.7 ;-0.61$ & 0 & 1,5708 \\
\hline \hline
\end{tabular}
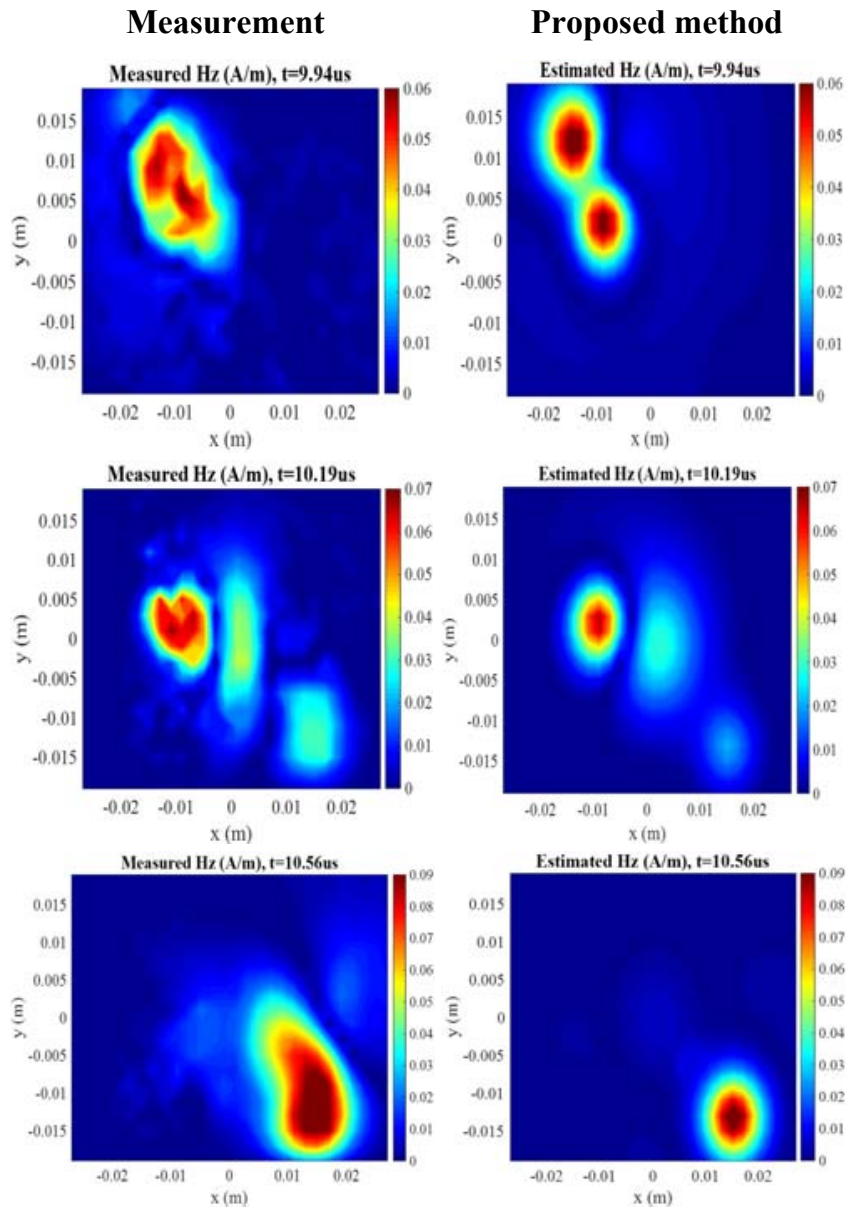

Fig. 14. Measured and estimated radiated field maps.

Looking at Figs. 14 and 15, at different time steps, we observe some dissimilarities in certain scanning points. In order to minimize differences between measured and estimated maps, we can refine the mesh of the emission zone and reduce even more the predefined error limit, defined in Fig. 2. However, this would require a considerable computation time and a large memory capacity. Nevertheless, the threshold value should not be less than measurements errors, otherwise we risk finding a model that does not correspond to reality (without a physical meaning). Hence, when the magnetic field value in the remaining map becomes inferior or equal to the magnitude of the measurements noise, the process of identifying a further source will stop. Indeed, the measurement error is the sum of the different errors that are due to coupling effects between probe and DUT, and to the NF test bench inaccuracies such as the uncertainties of the measuring device. In addition, errors are related to the spatial positioning of the measuring probe, the probe factor and the radius of the probe, which is very small (approximation of the field value in the whole area by the value in the center). Hence, we believe that the obtained model provides an efficient representative radiation behavior of the studied converter and that the equivalent magnetic dipoles are in good positions. Otherwise, using obtained results, it is possible to predict $H_{x}$ and $H_{y}$ components, which allows the validation of the obtained equivalent model. 

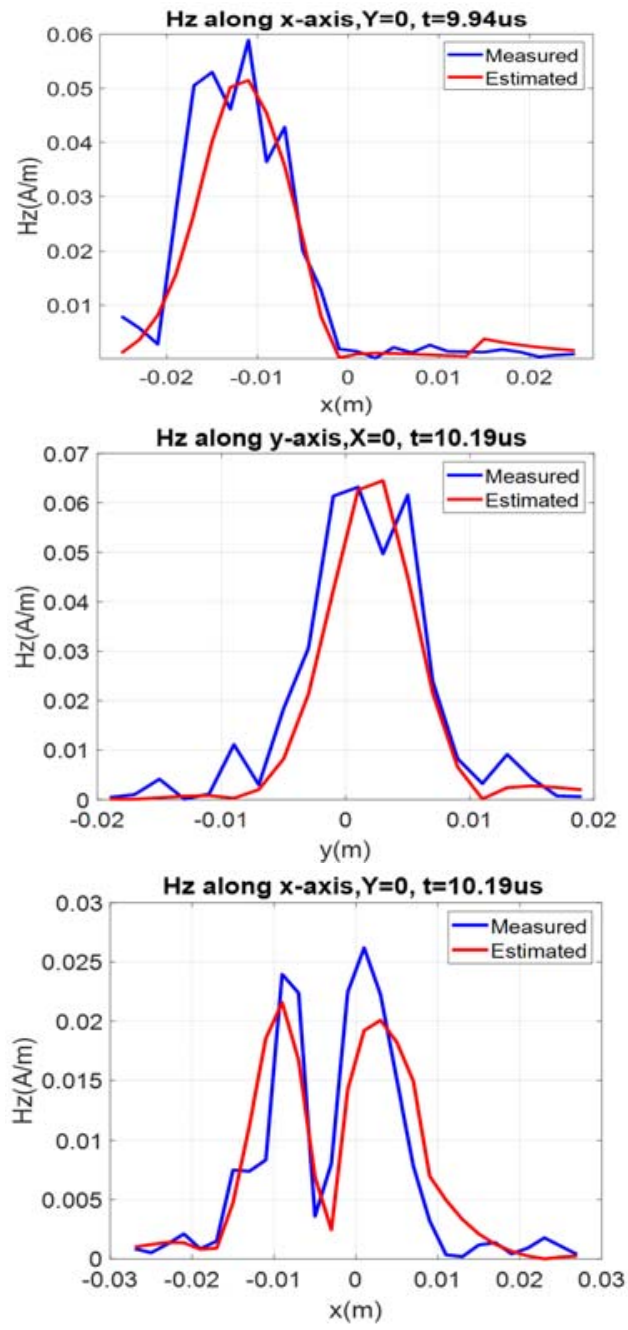

Fig. 15. Cuts of calculated and estimated $\mathrm{H}_{\mathrm{z}}$ at $Z=0$.

3) Validation of the proposed method using a conventional FD inverse problem based on Genetic Algorithms

In order to validate the proposed method, a comparative study between the EMTR-based method in the time domain and a conventional inverse method in the frequency domain has been performed. A fast Fourier transform of measured magnetic field signals is carried out in order to obtain the magnetic field distributions in the FD. Looking at the frequency spectrum of measured magnetic field at different positions, Fig. 16, we observe the presence of several harmonics over a frequency range that covers tens of megahertz. In order to acquire an equivalent model using the frequency inverse method, we have employed the standard GA as an optimization method [8-9], which is a widely adopted identification method in the literature. Therefore, the GA-based method has to be performed for several frequency components of the spectrum, which is a time consuming iterative process for both measurement and computations. Furthermore, GA options, such as population size and lower and upper variables bounds, have been properly chosen in order to accelerate the convergence of the method. Measured magnetic field distributions for different frequencies are shown in Fig. 17.

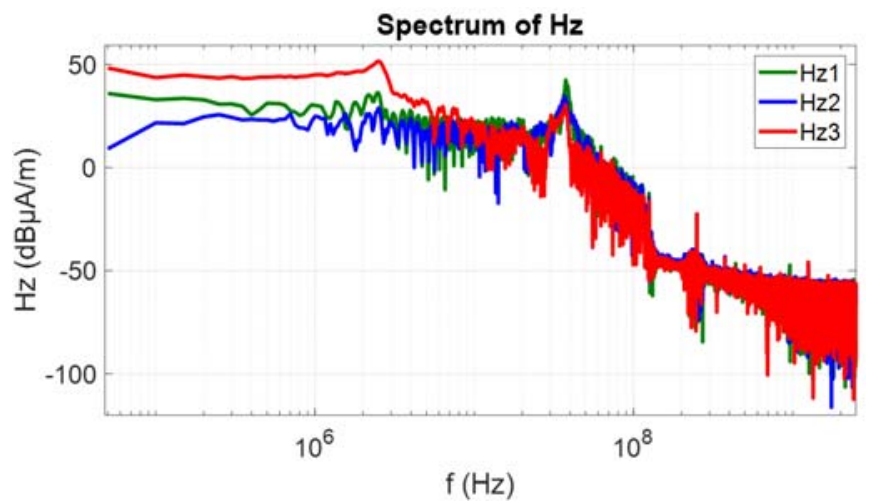

Fig. 16. Magnetic field spectrum measured above studied structure at different positions.
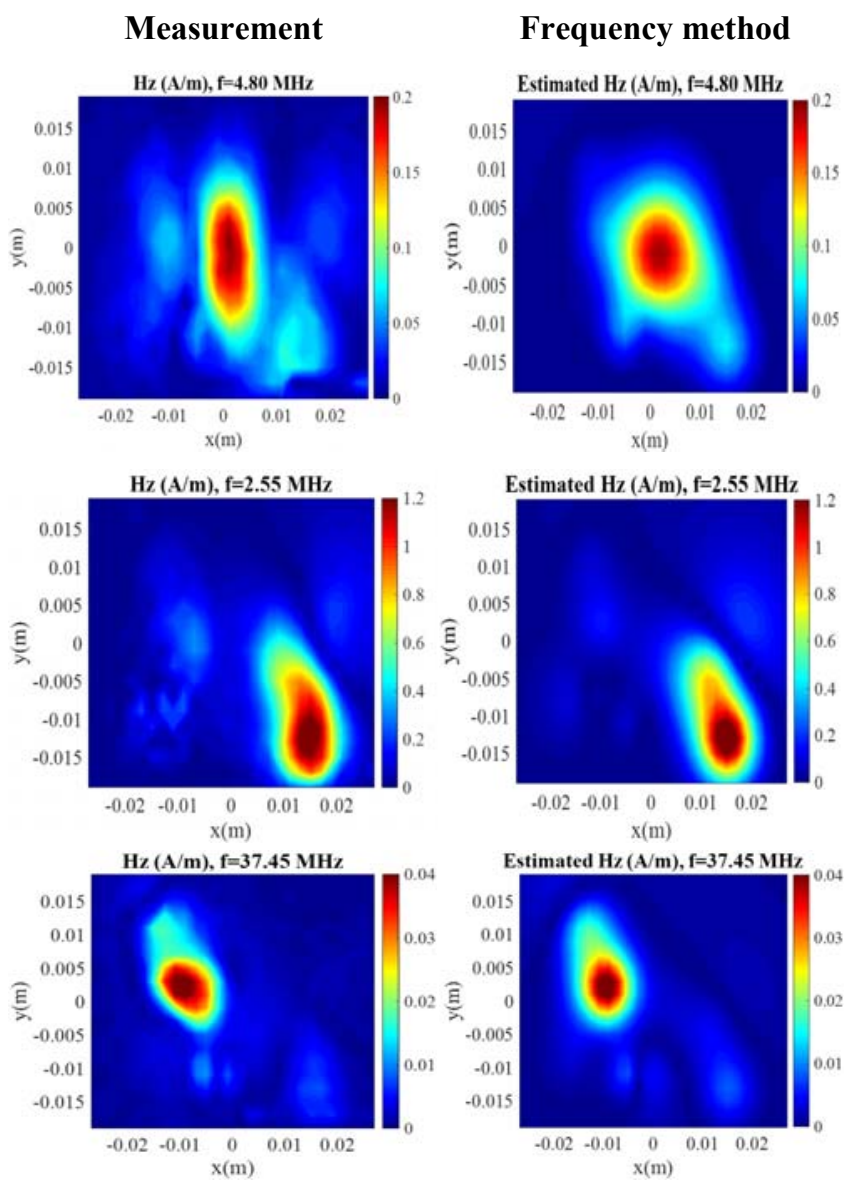

Fig. 17. Comparison of FD maps.

We notice the presence of the same equivalent dipoles using either FD or TD methods. The identified equivalent model is constituted of magnetic dipoles that have different radiation behaviors. Indeed, there is an adequate agreement between estimated and measured FD maps above studied structure, Fig. 17. Hence, this result confirms the obtained equivalent model using the EMTR-based method. It is worth noting that looking at the FD field distribution at a single frequency, we observe that some dipoles radiation contributions are absent. Therefore, magnetic field maps have to be measured and treated for several frequency components. Contrariwise, using TD-based method a 
full measurement acquisition is needed and the identification method is carried out through a single solve.

\section{CONCLUSION}

This paper is devoted to the investigation of NF radiated emissions characterization using time domain analysis. The proposed method is based on the inverse problem resolution to obtain an equivalent model that reproduces the same behavior as the radiating structure. In order to simulate radiated field, analytical expressions developed in time domain were used. The identification process is carried out using the time reversal technique. A TD test bench has provided an accurate measurement process able to identify EMC switching radiations around power electronic systems. Associated with a reasonable simulation time, the proposed method provides an adequate agreement between estimated and experimental results. For validation purposes, a comparative study between the EMTRbased method in the TD and a conventional inverse method based on the GA in the frequency domain has been performed.

The proposed methodwill have a great interest for application in EMC of advanced power electronic systems such as those developed for automotive lighting and renewable energy where non-sinusoidal EM radiations of short duration are emitted. Indeed, these kinds of power systems have a wide frequency band that cannot be analyzed only by FD methods. Our work shows that in case of transient signals having a wide frequency band, TR still produces focusing. The part of the field corresponding to higher frequencies appears to be sufficient to retrieve the position of the original source by time-reversal. Therefore, the proposed TD method will be an alternative method that can overcome FD insufficiency.

\section{REFERENCES}

[1] Paul, Clayton R. Introduction to electromagnetic compatibility. Vol. 184 John Wiley \& Sons, 2006.

[2] L. Beghou, F. Costa and L. Pichon, "Detection of Electromagnetic Radiations Sources at the Switching Time Scale Using an Inverse Problem-Based Resolution Method-Application to Power Electronic Circuits," in IEEE Transactions on Electromagnetic Compatibility, vol. 57, no. 1, pp. 52-60, Feb. 2015.

[3] Sivaraman, Nimisha. Design of magnetic probes for near field measurements and the development of algorithms for the prediction of EMC. Diss. Université Grenoble Alpes, 2017.

[4] Q. Chen, S. Kato, and K. Sawaya, "Estimation of current distribution on multilayer printed circuit board by near-field measurement," IEEE Trans. Electromagn. Compat., vol. 50, no. 2, pp. 399-405, May 2008.

[5] Y. Vives-Gilabert, C. Arcambal, A. Louis, F. de Daran, P. Eudeline and B. Mazari, "Modeling Magnetic Radiations of Electronic Circuits Using Near-Field Scanning Method," in IEEE Transactions on Electromagnetic Compatibility, vol. 49, no. 2, pp. 391-400, May 2007.

[6] Essakhi, B., et al. "Characterization of radiated emissions from power electronic devices: synthesis of an equivalent model from near-field measurement." The European Physical Journal-Applied Physics 38.3 (2007): 275-281.

[7] H. Shall, Z. Riah and M. Kadi, "A 3-D Near-Field Modeling Approach for Electromagnetic Interference Prediction," in IEEE Transactions on Electromagnetic Compatibility, vol. 56, no. 1, pp. 102-112, Feb. 2014.

[8] F. Benyoubi, L. Pichon, M. Bensetti, Y. Le Bihan and M. Feliachi, "An Efficient Method for Modeling the Magnetic Field Emissions of Power Electronic Equipment From Magnetic Near Field Measurements," in IEEE Transactions on Electromagnetic Compatibility, vol. 59, no. 2, pp. 609-617, April 2017.

[9] S. Saidi and J. Ben Hadj Slama, "A Near-Field Technique Based on PZMI, GA, and ANN: Application to Power Electronics Systems," in IEEE Transactions on Electromagnetic Compatibility, vol. 56, no. 4, pp. 784-791, Aug. 2014

[10] Ravelo, Blaise, and Yang Liu. "Computation of transient near-field radiated by electronic devices from frequency data." Fourier Transform Applications. InTech, 2012.

[11] B. Zitouna and J. Ben Hadj Slama, "Enhancement of Time-Domain Electromagnetic Inverse Method for Modeling Circuits Radiations," in IEEE Transactions on Electromagnetic Compatibility, vol. 58, no. 2, pp. 534-542, April 2016.

[12] Liu, Yang, and Blaise Ravelo. "Fully time-domain scanning of EM nearfield radiated by RF circuits." Progress in Electromagnetics Research, 57, (2014): 21-46.

[13] Liu, Yang, Blaise Ravelo, and Adam K. Jastrzebski. "Time-domain magnetic dipole model of PCB near-field emission." IEEE Transactions on Electromagnetic Compatibility 58.5 (2016): 1561-1569.

[14] B. Ravelo, Y. Liu and A. K. Jastrzebski, "PCB Near-Field Transient Emission Time-Domain Model," in IEEE Transactions on Electromagnetic Compatibility, vol. 57, no. 6, pp. 1320-1328, Dec. 2015.

[15] F. Rachidi, M. Rubinstein and M. Paolone, Electromagnetic Time Reversal: Application to Electromagnetic Compatibility and Power Systems, Wiley, 2017

[16] I. El Baba, S. Lallechere and P. Bonnet, "Electromagnetic Time-Reversal for reverberation chamber applications using FDTD," 2009 International Conference on Advances in Computational Tools for Engineering Applications, Zouk Mosbeh, 2009, pp. 157-162.

[17] M. Fink, "Time reversal of ultrasonic fields. I. Basic principles," in IEEE Transactions on Ultrasonics, Ferroelectrics, and Frequency Control, vol. 39, no. 5, pp. 555-566, Sept. 1992.

[18] J. Minonzio, M. Davy, J. de Rosny, C. Prada and M. Fink, "Theory of the Time-Reversal Operator for a Dielectric Cylinder Using Separate Transmit and Receive Arrays," in IEEE Transactions on Antennas and Propagation, vol. 57, no. 8, pp. 2331-2340, Aug. 2009. M.

[19] Conti, Stephane G., Philippe Roux, and William A. Kuperman. "Nearfield time-reversal amplification." The Journal of the Acoustical Society of America 121.6 (2007): 3602-3606.

[20] Z. Wang et al., "A Full-Scale Experimental Validation of Electromagnetic Time Reversal Applied to Locate Disturbances in Overhead Power Distribution Lines," in IEEE Transactions on Electromagnetic Compatibility, vol. 60, no. 5, pp. 1562-1570, Oct. 2018.

[21] M. Spirlet, V. Broun, P. Camus, C. Geuzaine, V. Beauvois and I. Molenberg, "Modelling time reversal applications in a reverberation chamber using the current image method," 2013 International Symposium on Electromagnetic Compatibility, Brugge, 2013, pp. 359364.

[22] P. Kosmas and C. M. Rappaport, "FDTD-based time reversal for microwave breast cancer detection-localization in three dimensions," in IEEE Transactions on Microwave Theory and Techniques, vol. 54, no. 4, pp. 1921-1927, June 2006.

[23] S. Hedia, B. Zitouna, J. Ben Hadj Slama and L. Pichon, "Electromagnetic Time Reversal for Radiating Source Identification in Time Domain," 2018 15th International Multi-Conference on Systems, Signals \& Devices (SSD), Yassmine Hammamet, Tunisia, 2018, pp. 531-536.

[24] S. Hedia, B. Zitouna, J. Ben Hadj Slama and L. Pichon, "A full time domain methodology based on near field time reversal for equivalent source identification," 2018 IEEE International Symposium on Electromagnetic Compatibility and 2018 IEEE Asia-Pacific Symposium on Electromagnetic Compatibility (EMC/APEMC), Singapore, 2018, pp. 141-146.

[25] El Baba, I., Lalléchère, S., \& Bonnet, P. (2012). Time Reversal for Electromagnetism: Applications in Electromagnetic Compatibility. Trends in Electromagnetism: From Fundamentals to Applications, 177.

[26] Ungureanu, A., Vuong, T. P., \& Ndagijimana, F. (2011). Electromagnetic Point Source Reconstruction by Reversed-TLM Method. Applied Computational Electromagnetics Society Journal, 26(9).

[27] Chabalko, M. J., \& Sample, A. P. (2016). Electromagnetic time reversal focusing of near field waves in metamaterials. Applied Physics Letters, 109(26), 263901. 
[28] J. De Rosny, G. Lerosey, and M. Fink, "Theory of electromagnetic timereversal mirrors," IEEE Trans. Antennas Propag., vol. 58, no. 10, pp. 3139-3149, Oct. 2010.

[29] De Rosny, J., \& Fink, M. (2007). Focusing properties of near-field time reversal. Physical Review A, 76(6), 065801.

[30] Jackson, John David. "Classical electrodynamics." (1999): 841-842.

[31] Balanis, Constantine A. "Antenna Theory: Analysis \& Design, John Willey \& Sons." Inc. Publication (1997).

[32] F. Benyoubi, M. Feliachi, Y. Le Bihan, M. Bensetti and L. Pichon, "Implementation of tools for electromagnetic compatibility studies in the near field," 2016 International Conference on Electrical Sciences and Technologies in Maghreb (CISTEM), Marrakech, 2016, pp. 1-6.

[33] B. Zitouna and J. Ben Hadj Slama, "Time domain inverse method based on the near field technique to solve electromagnetic interference problems: application to an AC/DC flyback converter," in IET Power Electronics, vol. 11, no. 13, pp. 2133-2139, 6112018. 